\title{
INFLUENCIA DEL GÉNERO EN LA SALUD DE LAS MUJERES CUIDADORAS FAMILIARES
}

\section{GENDER INFLUENCE IN THE HEALTH OF WOMEN FAMILY CAREGIVERS}

\section{Irma Fonseca Mardones ${ }^{1}$}

\begin{abstract}
RESUMEN
En Chile, cambios sociodemográficos y en el perfil epidemiológico han aumentado el número de personas que requieren de cuidados de salud, lo cual posiciona a el/la cuidador/a familiar como un rol que adquiere cada día mayor relevancia en nuestra sociedad. En nuestro país, la mayoría de los cuidadores familiares son mujeres.

El presente ensayo tiene como finalidad hacer una reflexión, desde un enfoque de género, acerca de cómo el ejercer el rol de cuidador/a familiar de una persona en situación de discapacidad puede afectar la salud de mujeres cuidadoras.

Para ello se discutirá acerca de cómo los mandatos de género condicionan la adquisición del rol de cuidadora familia y la forma en que éste se desempeña. Así también, se discutirá sobre cómo la articulación de los roles de cuidador familiar y mujer genera mayor sobrecarga en las mujeres, aumentando la posibilidad de comprometer su salud. Y finalmente, cómo los estereotipos de género pueden restringir que las mujeres cuidadoras familiares accedan a una atención de salud apropiada a sus necesidades, perpetuando de esta forma el compromiso a su salud y bienestar.

Los mandatos y estereotipos de género presionan a las mujeres a convertirse en cuidadoras familiares y definen la forma en que este rol debe ser desempeñado, lo que favorece el compromiso de la salud de las cuidadoras. Los estereotipos de género pueden influir en la calidad de la atención de salud que las cuidadoras reciben, contribuyendo al deterioro de su salud y calidad de vida.
\end{abstract}

\section{PALABRAS CLAVE}

Mujer, Cuidadora Familiar, Salud

\begin{abstract}
In Chile, sociodemographic changes and changes in the epidemiological profile, have increased the number of people who requiring health care, which positions the figure of the family caregiver as a role that becomes increasingly relevant in our society. In our country, most offamily caregivers are women.

The purpose of this essay is to reflect, from a gender perspective, on how exercising the role of family caregiver for a person with a disability, can affect the health offemale caregivers.

For this, it is discussed on how gender mandates conditions the acquisition of the family caregiver role and the way in which it performs; as well as it is discussed on how the articulation of the role of woman and family caregiver, generates greater overload in women, increasing the possibility of compromising their health. And finally, how gender stereotypes can restrict women family caregivers from accessing health care appropriate to their needs, thus perpetuating the commitment to their health and well-being.
\end{abstract}

1 Magíster en Ocupación y Terapia Ocupacional, Universidad de Chile. Diplomada en Docencia en Ciencias de la Salud. Universidad de Chile Terapeuta Ocupacional Hospital de la Dirección de Previsión de Carabineros de Chile. Departamento de Terapia Ocupacional y Ciencia de la Ocupación, Universidad de Chile. Vital Apoquindo 1200, Las Condes, Santiago. irma.fonseca@gmail.com. Fono: 229517275. https://orcid.org/0000-0002-9185-5631. 
Gender mandates and stereotypes pressure women to become family caregivers and define how this role should be performed, which favors the caregivers' commitment to health. Gender stereotypes can influence the quality of health care that caregivers receive, contributing to the deterioration of their health and quality of life.

\section{KEYWORDS}

Woman - caregivers - health

Recibido:10/11/2018

Aceptado: 03/09/2020

\section{INTRODUCCIÓN}

En Chile, las mejoras en la calidad de vida y en las condiciones sanitarias han generado cambios en el perfil epidemiológico y demográfico, provocando que en la actualidad las personas no sólo vivan más, sino que además, estén expuestas a sufrir una serie de enfermedades crónicas, aumentando el número de individuos que requieren cuidados ya sea por su edad o por estar en situación de discapacidad (Ministerio de Salud, 2010).

Frente a la necesidad de cuidados de salud surge la figura de el/la cuidador/a familiar (en adelante CF) como elemento clave que permite a las personas necesitadas de cuidado continuar viviendo en sus entornos cotidianos. Sin embargo, la prestación de cuidados implica para el/la CF una serie de efectos negativos que pueden llevar a comprometer su salud y calidad de vida.

Hace casi treinta años, Rodríguez afirmó que el cuidado familiar "se escribe en femenino singular" (Rodríguez, 1998 citado por García et al., 2004, s.p.) en una clara alusión a que este rol es desempeñado principalmente por mujeres y que en su ejecución cuentan con escasa ayuda de su entorno. Tradicionalmente, las mujeres han asumido dicho cuidado en acatamiento de mandatos de género según los cuales la función de la mujer es dar vida y cuidar de ésta.

El propósito de este ensayo es reflexionar acerca de la influencia del género en la salud de las mujeres $C F$ (en adelante MCF) de personas en situación de discapacidad (en adelante PsD) desde . Para esto se analizarán desde una perspectiva de género las características del cuidado familiar en salud, sus efectos en los/las CF, el rol de las mujeres en el cuidado familiar de salud, la dimensión pública del cuidado de salud de las mujeres y el compromiso de la salud de éstas como consecuencia del cuidado.

La perspectiva de género es un "instrumento de análisis que permite identificar las diferencias entre hombres y mujeres, para establecer acciones tendientes a promover situaciones de equidad" (Chávez, 2004, p.17), su empleo ha permitido comprender de una manera diferente los problemas, contribuyendo a delimitar con mayor claridad cómo la diferencia entre hombres y mujeres cobra dimensiones de desigualdad (Chávez, 2004).

\section{Cuidados familiares en salud}

El cuidado informal de salud ha sido definido como la "prestación de cuidados de salud a las personas que los necesitan por parte de familiares, amigos, vecinos $y$, en general, personas de la red social inmediata, que no reciben retribución económica por la ayuda que ofrecen" (Wright, 1983 citado por García et al., 2004, s.p.). En Chile, la mayoría de estos cuidados son proporcionados por la familia (Vaquiro \& Stiepovich, 2010).

Actualmente, se reconoce el beneficio de la permanencia de las PsD en sus hogares, (Jamieson, 1993 citado por Jofré \& Mendoza, 2005), por lo que los servicios sanitarios han puesto énfasis en la atención en el entorno de la persona, convirtiendo el hogar en un escenario de confluencia entre cuidados profesionales e informales (National Coordinating Group on Health Care Reform and Women, 2003 citado por García et al., 2004). Por otra parte, existe un vínculo entre los sistemas de cuidado formal e informal, en el cual la participación de 
los/las CF y la permanencia de la PsD en su hogar alivia la demanda asistencial y económica sobre el sistema formal de salud (Jofré \& Mendoza, 2005).

Si bien el cuidado informal corresponde a una empresa familiar (García, 2016), generalmente es el/la CF, quien asume la mayor parte del cuidado, siendo descrito/a como "la persona, habitualmente un familiar directo, que convive y mantiene la responsabilidad de proveer recursos que un paciente incapaz de auto sustentarse necesita" (Ballesteros, Martín, Ibarra, Loizaga et al., 2002 citado por Velásquez, López y Barreto, 2014, p. 68).

En general, cuando se habla de cuidado familiar en salud, éste se tiende a identificar con las acciones que el/la CF realiza directamente a la PsD. Sin embargo, se ha descrito que el cuidado familiar posee múltiples dimensiones que incluyen el cuidado directo, la monitorización e interpretación de lo observado, la toma de decisiones y las acciones subsiguientes, la obtención de recursos para el cuidado, la negociación con el sistema de salud, etc. (Schumacher, Stewart, Archbold, Dodd y Dibble, 2000 citado por Cuesta, 2009).

Las labores de cuidado se orquestan y desarrollan en función de las necesidades de la PsD, por lo que la vida $\mathrm{de}$ el/la CF se organiza en función de ésta, postergando sus propias necesidades e intereses, en una jornada sin principio ni fin (García et al., 2004).

La realización de estas labores y el desempeño de rol de $\mathrm{CF}$, adquieren para cada persona una forma y significado particular en base a su cultura y creencias, las que pueden constituirse como un elemento que ayude a sobrellevar la carga del cuidado o en su defecto a acentuarla (Pinquart y Sörensen, 2011 citado por Velásquez et al., 2014) .

Una vez asumido el rol de CF, el/la cuidador/a debe continuar ejerciendo sus otros roles en forma simultánea (García-Calvente et al., 2004), siendo la capacidad para mantener el equilibrio entre sus múltiples roles parte del desafío de convertirse y desempeñarse como CF. El conflicto entre los diferentes roles desempeñados (La Parra, 2001 citado por García, 2016) puede provocar que el/la CF comprometa su grado eficacia en el desempeño y/o nivel de participación en éstos, lo que puede traducirse en el abandono de actividades gratificantes y/o necesarias para el/la CF, dejando por ejemplo de recibir los beneficios de la interacción social o los beneficios económicos y previsionales de la actividad laboral (García et al., 2004).

Ciertas características del cuidado familiar afectan su visibilidad y reconocimiento social, como es el hecho de desarrollarse dentro del ámbito doméstico en forma no remunerada, en virtud de relaciones afectivas o de parentesco y ser realizado principalmente por mujeres, razones que han contribuido a que los/as CF sean un grupo social invisible (García, 2002 citado por García et al., 2004).

Se han descrito diferencias en cómo hombres y mujeres asumen el cuidado en relación a las tareas de las que se hacen cargo y del tiempo que les destinan. De acuerdo a esto, los hombres generalmente proporcionan menor cantidad de apoyo, manteniendo mayor distancia afectiva con la persona a quien cuidan y solicitan con mayor frecuencia asistencia de sus familiares (López, 2006). Al contrario, las mujeres generalmente asumen los cuidados más pesados relacionados con la asistencia en actividades de autocuidado e instrumentales, así como labores de acompañamiento y vigilancia, destinando más horas al día al cuidado en relación a los hombres (García et al., 2004; Metcalfe \& Padilla, 2019).

\section{Efectos del cuidado en el/la CF}

De acuerdo a la Organización Mundial de la Salud "la salud es un estado de completo bienestar físico, mental y social, y no solamente la ausencia de afecciones o enfermedades" (Organización Mundial de la Salud, 2014, p. 1). Salud y enfermedad son estados determinados por múltiples factores biológicos, psicológicos y sociales sin que exista entre ellos una línea clara que los separe, por lo que acontecimientos en cualquier nivel, desde el molecular al simbólico, repercuten en los demás niveles (Lipowski, 1988 citado por IzquierdoSotorrío, 2011). Así, se entiende que compromisos en una dimensión tienen el potencial de impactar en otros aspectos de la persona, generando un compromiso en su salud y calidad de vida.

En términos ocupacionales, asumir el rol de CF implica un aumento en las demandas ocupacionales que provoca desequilibrio ocupacional en el/la cuidador/a, frente al cual, éste/a debe responder desarrollando nuevas habilidades, destrezas y rutinas. En la medida que las demandas continúen sobrepasando las capacidades 
de el/la CF la situación de desequilibrio ocupacional se mantendrá, teniendo el potencial de provocar un compromiso, temporal o permanente, en su salud y bienestar.

En la literatura, múltiples estudios han señalado que el cuidado familiar en salud tiene un impacto negativo, tanto en la salud física como emocional, de el/la CF (Estrada et al., 2018; Montoya et al., 2018; Yepes et al., 2018; Metcalfe \& Padilla, 2019). Los efectos del cuidado no se reducen sólo al desgaste producto de las labores de cuidado, también incluyen el impacto afectivo surgido por el cuidado y por el duelo producido por la discapacidad (Corominas, 2001, Bowlby, 1976 citados por Urbano y Yuni, 2008). También se agrega la pérdida de beneficios derivados de la participación en otras áreas de desempeño ocupacional importantes para la persona y el escaso reconocimiento a nivel social del rol de CF.

A lo anterior se agrega que los/as CF tienden postergar su autocuidado en función del cuidado y de la PsD (Velásquez et al., 2014). Estudios demuestran que los/as CF tienden a recurrir menos a los servicios sanitarios, en comparación con el resto de la población (Bover, 2004), lo que puede contribuir a aumentar el compromiso de su salud.

\section{Mujer y cuidado familiar en salud}

Las labores de cuidado en salud han sido tradicionalmente adscritas a las mujeres (Yepes et al., 2018), siendo mujeres la mayoría de las CF (Herrero, 2017; GonzálezSolanellas et al., 2018; Yepes et al., 2018).

Según el mandato patriarcal imperante, la función de las mujeres es "dar la vida, protegerla, cuidarla, reproducirla y mantener a personas concretas en las mejores condiciones posibles" (Lagarde, 1999, p. 20). De acuerdo a esto, lo más importante en la vida de las mujeres es "cuidar y seguir cuidando, y volver a cuidar a otras y otros, toda la vida" (Lagarde, 1999, p. 20).

Considerado como una extensión de los cuidados proporcionados a los miembros de la familia, las labores de cuidado en salud forman parte del rol femenino, comprenden el garantizar un ambiente seguro y acorde a las necesidades de cada miembro de la familia
(Cardaci, 1992 citado por Jofré y Mendoza, 2005). Para esto, las mujeres estarían dotadas de cualidades como ternura, dulzura, debilidad, emotividad, instinto maternal, etc. (Fernández, 2001) lo que las hace idóneas para estas tareas.

Este mandato de género del cuidado, no solo determina la presión hacia la mujer para asumir el cuidado, si no también, mandata la forma en que dicho cuidado debe ser realizado, esto es, poniendo el foco de sus vidas en los otros/as y no en ellas mismas, implicando así, una permanente postergación, subordinando constantemente su propia salud al bienestar de los demás (Jofré \& Mendoza, 2005).

Los mandatos y estereotipos de género prescriben, no solo la forma en que los miembros de una determinada cultura ven a las mujeres y su rol en la sociedad, sino también, la forma en que las mujeres se ven a sí mismas (Botello \& Casado, 2016), cómo ven a los otros/as y las conductas que esperan de ellos/as (Hermosa \& Mejía, s. f.). De esta forma, los mandatos y estereotipos de género contribuyen a definir las expectativas externas e internas del rol de MCF, fundiendo así las expectativas de dos roles, mujer y CF.

De igual forma, también es posible que el rol de MCF se superpone con otros roles desempeñados en forma simultánea, como el de madre, esposa, etc. (Yepes et al., 2018), por lo que el desempeño del rol de MCF puede ser evaluado en más de un nivel, aumentado así la presión sobre la mujer al implicar la valoración de su desempeño en otros roles significativos.

Por su fuerte carga cultural, el incumplimiento de las expectativas de rol basadas en estereotipos de género puede considerarse como una transgresión al orden natural de las cosas, pudiendo ser sometida a un doble enjuiciamiento: a nivel social (expectativas externas) y a nivel de la propia persona que las infringe (expectativas internas). Lo anterior, puede generar insatisfacción con el desempeño del rol, y exponer a la MCF a sanciones, tanto externas como internas, producto de un desempeño de rol considerado como inadecuado. Estas sanciones pueden adquirir diversas formas de acuerdo a la naturaleza de sus acciones, sus características y creencias personales, las características socioculturales y económicas de su entorno y el tipo de patriarcado al que esté sometida. 
Por otra parte, en la actualidad, cambios experimentados a nivel social y económico han contribuido al aumento de la carga de las mujeres en general, incluidas las MCF. En Chile, el aumento de mujeres jefas de hogar (Ministerio del Trabajo y Previsión Social, 2016) ha generado mayor demanda familiar, social y económica sobre este grupo de mujeres. La creciente incorporación de las mujeres al mercado laboral, caracterizado por bajos sueldos y escasa flexibilidad laboral (Pla et al., 2013). A lo anterior, se agrega el hecho de que producto de las políticas de planificación urbana de las ciudades se ha producido un crecimiento de éstas hacia zonas periféricas (De Mattos, Fuentes \& Link, 2014), generando que los lugares de trabajo se encuentran alejados de los hogares, aumentando el período en que las mujeres permanecen fuera de su hogar a causa del trabajo. Condiciones que en su conjunto, contribuyen a generar una mayor vulnerabilidad en las MCF.

Investigaciones demuestran que las mujeres de menores ingresos económicos tienden a asumir con mayor frecuencia el rol de CF en comparación con las de mayores ingresos, quienes cuentan con más recursos para contratar servicios de cuidado (Jofré \& Mendoza, 2005; INSERSO, 1995; La Parra, 2001 citado por García et al., 2004). Lo cual contribuye acentuar la situación de vulnerabilidad de las MCF al aumentar el número de condicionantes sociales desfavorables.

De esta forma, el rol ya de por sí extenuante y potencialmente riesgoso para la salud de CF, adquiere elementos aún más demandantes cuando es desempeñado por mujeres, debido a la condición de vulnerabilidad en las que éstas se encuentran, determinada por factores sociales, culturales y económicos que ponen sobre ellas una mayor carga de trabajo, menor retribución económica y reconocimiento social por éste. A lo anterior, se agrega que, si bien las mujeres tienen más posibilidades de ser CF, tienen menos posibilidades de ser cuidadas por sus familias (Rodríguez \& Sancho, 1999 citado por Crespo \& López, 2007), lo que contribuye a favorecer su condición de vulnerabilidad futura.

\section{Dimensión pública del cuidado en salud de las mujeres}

Las acciones privadas tienen una repercusión pública y los actos y decisiones públicos tienen un impacto en lo privado, de forma que decisiones a nivel macro impactan en lo más íntimo de nuestra cotidianidad y viceversa. De acuerdo a esto, es posible ver como estrategias de reducción de costos en salud emanadas de políticas neoliberales conllevan un desplazamiento de la prestación de cuidados de salud desde el sistema formal hacia el informal, impactando en la vida cotidiana de miles de mujeres.

Estas decisiones se apoyan en la convicción de que es posible disponer del tiempo y el trabajo gratuito de las mujeres y en la obligación moral de éstas de cuidar a otros (Gómez, 2002). Es esta gratuidad e informalidad de la no-relación entre Estado y MCF la que libera al primero, no sólo de obligaciones salariales, sino también de obligaciones asociadas a la mantención de su salud, condiciones de trabajo, beneficios previsionales y, aún más, a reconocerlas como participantes relevantes de las políticas de salud.

La participación en la producción social de la salud posee una doble dimensión, por un lado, está la carga de trabajo y por otro, la distribución de los beneficios, el reconocimiento y el poder. En Chile, las MCF de PsD representan el 73,9\% de los cuidadores (Ministerio de Desarrollo Social, 2015), sin embargo, el limitado reconocimiento que el Estado hace de su labor se traduce en una escases de políticas públicas que las contemplen. Actualmente, sólo podemos mencionar el derecho de el/la CF de una PsD de ausentarse del trabajo en caso de enfermedad de ésta, por un máximo de go horas al año, las que deben ser restituidas con trabajo efectivo o descontadas del feriado legal (Ministerio de Desarrollo Social y Familia, 2020). Y el Programa de atención domiciliaria a personas con dependencia severa (Ministerio de Salud de Chile, 2007) que contempla prestación de servicios y remuneración económica a el/la CF, dirigido a personas con alto grado de dependencia, por lo que deja fuera de su cobertura a un enorme número de CF.

Por otra parte, las mujeres suelen estar subrepresentadas en las estructuras de poder que definen las prioridades y asignan los recursos, lo que las pone en una posición de desventaja sistemática dentro del sistema (Gómez, 2002).

De acuerdo a Nieves y Robles (2016), el cuidado es un bien público esencial para el funcionamiento de las sociedades, un derecho fundamental y una necesidad en diversos momentos del ciclo vital. No obstante lo anterior, a pesar de que en los últimos años se 
han implementado una serie de estrategias dirigidas a reconocer la labor de las MCF en distintos ámbitos del cuidado (Nieves \& Robles, 2016), el Estado y los sistemas de salud públicos y privados, continúan beneficiándose del trabajo de las mujeres sin compensarlas apropiadamente, ni hacerse cargo del impacto que el cuidado genera en sus vidas.

\section{Efectos del cuidado: la mujer se enferma}

En respuesta a la presión social y a sus propias creencias acerca de su rol en la sociedad, las mujeres se convierten en $\mathrm{CF}$, hecho que dadas las características de este rol y de los significados personales asociados a él, sumado a las múltiples demandas a las que las mujeres se ven sometidas en los distintos ámbitos de su vida, genera un impacto negativo en su salud y calidad de vida.

Estudios afirman que la salud de las mujeres se afecta más que la de los hombres producto de ser CF, (García et al., 2011; Estrada et al., 2018; Metcalfe \& Padilla, 2019). Algunos autores atribuyen este mayor impacto en la salud, entre otras cosas, al hecho de que las mujeres utilizan estrategias de afrontamiento menos efectivas que las empleadas por los hombres (Artaso, Goñi \& Gómez, 2001a, Lutzky \& Knight, 1994, Rose-Rego et al., 1998 citado por López, 2006; Thompson, Sugiyama y Bell S et al., 2004; Thoits, 1995 citado por Velásquez et al., 2014), o a una peor utilización de mecanismos de amortiguación del estrés (Larrañaga et al., 2009).

Lo anterior no deja de llamar la atención, puesto que, de acuerdo a los estereotipos de género, son la naturaleza y características propias de las mujeres, las que las hacen más idóneas para el cuidado. Frente a lo cual cabe preguntarse, ¿ es razonable que los motivos en los que se basa la distribución por género de las labores de cuidado sean también la causa de la mayor afectación de la salud de las mujeres? Y si esto es cierto, ¿por qué entonces no son los hombres quienes asumen los cuidados, ya que a la luz de dichos estudios pareciera ser que están mejor capacitados para ello? Si se sabe que las mujeres se enferman más a causa del cuidado ¿no es la presión a asumir el cuidado un acto deliberado de perjuicio hacia ellas?

Lo cierto es que la asimetría en el compromiso a la salud puede explicarse en base al tipo y naturaleza de los cuidados proporcionados por hombres y mujeres, en los que ellas asumen las tareas más pesadas durante más horas al día y reciben menos ayuda de su entorno, a la mayor carga de trabajo que las mujeres asumen en general y a que las MCF tienden a postergar sus necesidades, desatendiendo, entre otras cosas su autocuidado (Jofré \& Mendoza, 2005), así como, a la condición de vulnerabilidad en la que las mujeres se encuentran en distintos ámbitos. Cuando la carga del cuidado se reparte equitativamente entre ambos sexos la diferencia en el compromiso de la salud disminuye o desaparece (Manaset \& La Parra, 2011; Larrañaga et al. 2008 citado por Pérez \& Morales, 2014).

El intento de traspasar la responsabilidad del mayor compromiso en la salud de las MCF a las propias mujeres, no hace sino minimizar sus capacidades y desvalorizar su labor, reforzando una posición de inferioridad en relación a los hombres. Puesto que la participación de las mujeres en su autocuidado se sustenta en la "facultad de reconocerse a sí mismas como personas, de valorarse y reivindicar el poder de decidir sobre su salud" (Arango, 1992 citado por Jofré \& Mendoza, 2005, p. 40), la invisibilización y desvalorización social del rol de MCF pueden influir en la falta de atención de éstas frente al deterioro de su salud.

Por otra parte, la articulación del rol de CF y mujer, no sólo favorece que las MCF comprometan su salud, sino que también, puede constituirse como una barrera en la obtención de una apropiada atención de salud.

Los estereotipos de género son construcciones sociales compartidas en mayor o menor medida por todos los miembros de una cultura, incluidos los profesionales de la salud, dando origen a lo que se denomina sesgos de género en atención de salud (Pla, Adam, \& Bernabeu, 2013; Ruiz-Cantero et al., 2019). Estos corresponden a la tendencia a la concepción y la praxis médicas por y para un sujeto normativo hegemónico. Se pueden expresar en la universalización de abordajes diagnóstico-terapéutico-preventivos basados en la extrapolación de resultados de investigación en sujetos próximos al normativo hegemónico, o, en un abordaje diferencial basado en creencias y/o estereotipos y no en evidencia científica (Tasa-Vinyals, Mora \& Raich, 2015; Aizenberg \& Maure, 2017; Herrero, 2017).

Así, aunque las mujeres acuden con mayor frecuencia a los servicios generales de salud (Pla et al., 2013), sus manifestaciones clínicas tienden a ser consideradas 
menos creíbles que las de los hombres (Pla et al., 2013; Herrero, 2017 ). Al mismo tiempo, la escasez de investigaciones centradas en las necesidades integrales de las mujeres puede generar abordajes limitados a sus problemáticas de salud (Botello \& Casado, 2016). Lo anterior puede contribuir a que sus manifestaciones clínicas sean erróneamente interpretadas y que la atención de salud recibida resulte inadecuada a sus necesidades (Quevedo, 2017), provocando que permanezcan enfermas o que su salud se comprometa aún más.

\section{El cuidado familiar... ¿se escribe en femenino singular?}

Hace más de veinte años Rodríguez afirmó que el cuidado familiar "se escribe en femenino singular" (Rodríguez, 1998 citado por García et al., 2004, s.p.) en alusión al hecho de que éste es desarrollado mayoritariamente por mujeres y que en su desempeño ellas cuentan con escasa o nula ayuda de su entorno.

De acuerdo a la Real Academia Española, dentro de los significados de la palabra "escribir" están: "representar las palabras o las ideas con letras u otros signos trazados en papel u otra superficie; componer libros, discursos; comunicar a alguien por escrito algo; trazar las notas y demás signos de la música; inscribirse en una lista de nombres para un fin", etc. (Real Academia Española, 2014 , s.p.). Ante lo cual podemos reflexionar, ¿̇realmente es posible considerar que son las mujeres quienes han trazado el rol de CF? o más bien, ¿éste corresponde a un rol escrito para ellas por estructuras patriarcales muchos años atrás y reeditado periódicamente?, cuando las mujeres desempeñan el rol de $\mathrm{CF}_{\text {¿qué tanto de }}$ las acciones, labores y significados atribuidos nacen de ellas mismas y cuanto es emanado de mandatos y estereotipos de género asumidos como naturales?, ¿son las mujeres realmente libres para decidir inscribirse en la larga lista de $\mathrm{CF}$ ?

Al respecto podemos dar una mirada distinta a la frase de Rodríguez. El cuidado familiar no "se escribe en femenino singular", sino que más bien, históricamente se ha escrito en masculino plural. Han sido los hombres, quienes han prescrito la asignación del rol de CF a las mujeres, mandatando a su vez, la forma en que éste debe ser desempeñado. Frente a lo cual, las mujeres se han visto compelidas a desempeñar dicho rol en múltiples femeninos singulares, los que dadas las características de aislamiento, invisibilidad y falta de recursos en el cual permanecen, les ha impedido adquirir conciencia de su derecho de tener derechos (Galheigo, 2007 citado por Galheigo, 2012), impidiendo que lleguen a articularse en un plural, que les permita constituirse en un colectivo, de forma de alcanzar los beneficios de la asociatividad en la visibilización y lucha por sus reivindicaciones.

Lo cierto, es que es difícil decir que convertirse en MCF represente un ejercicio de autonomía por parte de las mujeres. Resulta imperioso reconocer que esto es promovido por estructuras de poder que se ven favorecidas económica y socialmente por el trabajo gratuito de las mujeres, manteniéndolas confinadas al ámbito doméstico, sin tiempo para contactarse con otros/as, invisibles tras los muros de sus casas, sin tiempo para perseguir sus propios intereses, enfermas o lo suficientemente enfermas para comprometer su bienestar y potencial, pero no para evitar que continúen cuidando.

\section{Conclusiones}

Los mandatos y estereotipos de género presionan a las mujeres a convertirse en CF y definen cómo este rol debe ser desempeñado.

La forma en que está prescrito el rol de MCF favorece el deterioro de la salud de las cuidadoras. Los estereotipos de género pueden influir en la calidad de la atención de salud que las MCF reciben, contribuyendo al detrimento de su salud y calidad de vida.

Al fusionarse los roles de mujery $\mathrm{CF}$, los mandatos y estereotipos de género contribuyen a delinear las expectativas internas y externas del rol de MCF. De acuerdo a esto, la falla en el cumplimiento de estas expectativas puede afectar la satisfacción con el desempeño de ambos roles en forma simultánea, afectando así diversas áreas de desempeño de las MCF. Con lo cual, tienen el potencial de amplificar sus efectos negativos sobre su salud y calidad de vida.

Cuidar de las PsD representa una responsabilidad ética de la sociedad y de las familias hacia sus miembros, no sólo es una muestra de afecto, sino que es parte del ejercicio de las personas en su calidad de ciudadanos de contribuir a la sociedad y al bienestar común. 
En respuesta a la pregunta inicial que motiva este ensayo, podemos decir que el género no sólo influye en la forma en que las mujeres se ven a sí mismas, sino también en cómo la sociedad las ve y lo que espera de ellas, influye en su decisión convertirse en CF y la forma en que ejecutan este rol, lo que favorece que comprometan su salud. A la vez el género, influye en la calidad de la atención de salud que las mujeres reciben, atención inadecuada que puede provocar que ellas continúen enfermas, agravando de esta forma aún más la situación de vulnerabilidad en la cual viven.

Si bien el presente ensayo se ha enfocado en realizar un análisis de la situación de las MCF y los efectos de dicho rol en su salud desde un enfoque de género, resulta evidente que la situación vivida por las MCF corresponde a una problemática intersectorial en la que concurren elementos socioculturales, de género y económicos, por lo que cualquier estrategia dirigida a su abordaje debe considerar todos estos elementos.

\section{RefERENCIAS BibLIOGRÁFICAS}

Aizenberg, L., \& Maure, G. (2017). Migración, salud y género: Abordajes de proveedores de salud en la atención de mujeres migrantes bolivianas en la provincia de Mendoza, Argentina. REMHU: Revista Interdisciplinar da Mobilidade Humana, 25(51), 149-164. https://doi.org/10.1590/1980-85852503880005110

Botello, A. \& Casado, R. (2016). Estereotipos de género con respecto a las etapas reproductivas de las mujeres y sus implicaciones en la salud. Matronas Profesión, 17(4), 130-136.Cuesta Benjumea, C. de la. (2009). El cuidado familiar: una revisión crítica. Investigación y educación en enfermería, 27(1), 96-102.

Bover, A. (2004). Cuidadores informales de salud del ámbito domiciliario: percepciones y estrategias de cuidado ligadas al género ya la generación. Universitat de les Illes Balears.

Crespo, M., \& López, J. (2007). El apoyo a los cuidadores de familiares dependientes en el hogar: desarrollo del programa ¿cómo mantener su bienestar? $\left(1^{\circ}\right)$. Madrid: Ministerio de Trabajo y asuntos Sociales.

Cuesta, C. de la. (2009). El cuidado familiar: una revisión crítica. Investigación y educación en enfermería, 27(1), 96-102

Chávez, J. (2004). Perspectiva de Género. $1^{\circ}$ Edición. Plaza y Valdés S.A. de C.V., México

De Mattos, C., Fuentes, L., \& Link, F. (2014). Tendencias recientes del crecimiento metropolitano en Santiago de Chile:?' Hacia una nueva geografía urbana? Revista invi, 29(81), 193-219.

Esteban, A. (2016) Revisión bibliográfica: Impacto de los estereotipos de género en la vida diaria de las mujeres. Un análisis desde la justicia ocupacional. TOG (A Coruña) (revista en Internet) (con- sultada en 8 de diciembre de 2017); 13(24): (21p.). Disponible en: http://www.revistatog.com/num24/pdfs/revision3.pdf

Estrada, M. E., Gil, A. I., Gil, M., \& Viñas, A. (2018). La dependencia: Efectos en la salud familiar. Atención Primaria, 50(1), 23-34. https:// doi.org/10.1016/j.aprim.2016.12.007

Fernández, E. A. (2001). La socialización de género a través de la programación infantil de televisión. Valladolid, España en, http://www. fyl. uva. es/wceg/articulos/TelevisionPatriarcal. pdf(consultado el 27/01/2012). Recuperado a partir de http://cdd.emakumeak. org/ficheros/0000/0383/TelevisionPatriarcal.pdf

García, M., del Río, M., \& Marcos, J. (2011). Desigualdades de género en el deterioro de la salud como consecuencia del cuidado informal en España. Gaceta Sanitaria, 25, 100-107. https://doi. org/10.1016/j.gaceta.2011.09.006

García, E. P. L. (2016). Cuidar a los que cuidan. Ciencia y Salud Virtual, $8(2), 62-70$.

Gómez, E. (2002). Equidad, género y salud: retos para la acción. Revista Panamericana de Salud Pública, 11 (5/6), 454-461.

González-Solanellas, M., Puig, M., Rodriguez, P., \& Zabaleta-delOlmo, E. (2018). Estudio prospectivo de la incidencia de eventos cardiovasculares en adultos jóvenes y su relación con el síndrome metabólico. Atención Primaria, 50(1), 67-69. https:// doi.org/10.1016/j.aprim.2017.01.016

Herrero, M. S. (2017). Salud mental, género e igualdad. Norte de Salud mental, XIV no56, 109-114

Izquierdo-Sotorrío, E. (2015).Los mecanismos de defensa desde la perspectiva de género y su impacto sobre la salud. Revista Digital de Medicina Psicosomática y Psicoterapia, 1(5), 1-27

Jofré, A., \& Mendoza, P. (2005). Toma de decisiones en salud en mujeres cuidadoras informales. Ciencia yenfermería, 17(1), 37-49.

Lagarde, M. (1999). Claves feministas para el poderío y a autonomía de las mujeres. Puntos de Encuentro. Sevilla: Instituto Andaluz de la Mujer.

Lagarde, M.. (2005). Los cautiverios de la mujeres. Madresposas, monjas, putas, presas y locas $\left(4^{\circ}\right)$. Universidad Autónoma de México.

Larrañaga, I., Valderrama, M. J., Martín, U., Begiristain, J. M., Bacigalupe, A., \& Arregi, B. (2009). Mujeres y hombres ante el cuidado informal: diferencias en los significados y las estrategias. Revista Facultad Nacional de Salud Pública, 27(1), 50-55.

López, M., Orueta, R., Gómez-Caro, S., Sánchez, A., Carmona, J., Moreno, A., \& Javier, F. (2009). El rol de cuidador de personas dependientes y sus repercusiones sobre su calidad de vida y su salud. Revista Clínica de Medicina de Familia, 2(7), 332-339.

López, J. (2006). Entrenamiento en manejo del estrés en cuidadores de familiares mayores dependientes: desarrollo y evaluación de la eficacia de un programa : memoria para optar al grado de doctor. [Universidad Complutense], Servicio de Publicaciones, Madrid. Metcalfe, I. M., \& Padilla, I. L. (2019). Desigualdades de género en los impactos del cuidado informal de mayores dependientes en Gipuzkoa: Estudio CUIDAR-SE. Rev Esp Salud Pública., 93, 1-13. 
Ministerio de Desarrollo Social de Chile (2015) II Estudio Nacional de la Discapacidad, Santiago, Chile. Recuperado de https://www. google.com/search?client=safari\&rls=en\&q=Ministerio + de+De sarrollo+Social+(2015)+Il+Estudio+Nacional+de+la+Discapacida $\mathrm{d},+$ Santiago, + Chile\&ie $=$ UTF-8\&oe=UTF-8

Ministerio de Desarrollo Social y Familia de Chile (2020) Permisos a Padres y Cuidadores. Recuperado de https://www.senadis.gob. $\mathrm{cl} /$ pag/377/734/preguntas_frencuentes

Ministerio de Salud de Chile (2007). Programa de Atención Domiciliaria A personas con Dependencia severa, 1-13

Ministerio de Salud de Chile (2010). Estrategia nacional de salud para el cumplimiento de los objetivos sanitarios de la década 2011-2020. Santiago, Chile: MINSAL

Montoya, D. I. G., Machado, S. Z., \& Gómez, V. U. (2018). Sobrecarga en los cuidadores principales de pacientes con dependencia permanente en el ámbito ambulatorio. Medicina U.P.B., 37(2), 1-9.

Organización Mundial de la salud. (2014). Documentos Básicos. $48^{\circ}$ Edición. Ediciones de la OMS. https://apps.who.int/gb/bd/ PDF/bd48/basic-documents-48th-edition-sp.pdf

Pérez, M. \& Morales J. (2014) Desigualdades de género en el cuidado de grandes dependientes y su impacto en la salud. Revista Cuidándote Digital, VII (2): 1 - 16

Pla, I., Adam, A., \& Bernabeu, I. B. (2013). Estereotipos y prejuicios de género: factores determinantes en Salud Mental. Norte de Salud mental, 17(46), 20-28.

Quevedo, C. (2017). Perspectiva de género en la salud de la mujer. Universidad de Cantabria. Cantabria, España

Ramírez-Perdomo, C., Salazar-Parra., Y., Perdomo-Romero, A. (2017) calidad de vida de cuidadores de personas con secuelas de trastornos neurológicos. Enfermería Neurológica, 45(C): 9 -16

Real Academia Española y Asociación de Academias de la Lengua Española (2014). «escribir». Diccionario de la lengua española (23. ${ }^{\mathrm{a}}$ edición). Madrid: Espasa. ISBN 978-84-670-4189-7. Consultado el 9 de diciembre de 2017

Ruiz-Cantero, M. T., Tomás-Aznar, C., Rodríguez-Jaume, M. J., PérezSedeño, E., \& Gasch-Gallén, Á. (2019). Agenda de género en la formación en ciencias de la salud: Experiencias internacionales para reducir tiempos en España. Gaceta Sanitaria, 33(5), 485-490. https://doi.org/10.1016/j.gaceta.2018.03.010

Tasa-Vinyals, E., Mora, M., \& Raich, R. M. (2015). Diseño de un sistema para el estudio del sesgo de género en la praxis médica en atención primaria mediante situaciones clínicas modelo.Jornada d'Atenció Primària al Món Rural.

Urbano, C., \& Yuni, J. (2008). Discapacidad en la escena familiar ( $1^{\circ}$ Edición). Argentina: Editorial Encuentro.

Vaquiro, S. \& Stiepovich, J. (2010) Cuidado informal: un reto asumido por la mujer. Ciencia y enfermería, XVI (2): 9 -16

Velásquez, V. F., López, L., \& Barreto, Y. (2014). Cuidadores familiares campesinos: carga de cuidado, tiempo de cuidado y grado de funcionalidad. Investigación en Enfermería: Imagen y Desarrollo, 16(2), 65. https://doi.org/10.11144/Javeriana.IE16-2.cfcc

Yepes, C. E., Arango R., A. L., Salazar, A., Arango, E. M., Jaramillo, A. L., Mora, J. E., \& Posada Zapata, I. C. (2018). El oficio de cuidar a otro: "Cuando mi cuerpo está aquí pero mi mente en otro lado". Revista Ciencias de la Salud, 16(2), 294. https://doi.org/10.12804/ revistas.urosario.edu.co/revsalud/a.6771 
